\title{
Förster Energy Transfer in Thin Films of Conjugated Polymers and in Solution
}

\author{
Arnab Mukherjee and Biman Bagchi* \\ Solid State and Structural Chemistry Unit, Indian Institute of Science, Bangalore 560012, India
}

\begin{abstract}
Fluorescence resonance energy transfer (FRET) is routinely used to study equilibrium and dynamical properties of polymers and biopolymers in the condensed phases. It is now being widely used in conjunction with single molecule spectroscopy where one usually employs the Förster expression which predicts $\left(1 / R^{6}\right)$ distance dependence of the energy transfer rate. However, critical analysis shows that this expression can be of rather limited validity in many cases. We demonstrate this by explicitly considering a donoracceptor system, polyfluorene $\left(\mathrm{PF}_{6}\right)$-tetraphenylporphyrin (TPP), where their sizes are comparable to the distance separating them. In such cases, one may expect much weaker distance (as $1 / \mathrm{R}^{2}$ or even weaker) dependence. We also discuss recent application of FRET to study biopolymer conformational dynamics. We show that the well-known Wilemski-Fixman theory needs to be generalized to treat FRET at short separations. The utility of FRET in studying protein folding is also discussed.
\end{abstract}

Keywords: Fluorescence resonance energy transfer (FRET); Förster expression; Polymer dynamics; Protein folding.

\section{INTRODUCTION}

Fluorescence resonance energy transfer (FRET) is a highly popular tool to study equilibrium and dynamical properties of polymers and biopolymers in condensed phases. The rate of FRET is usually assumed to be given by the Förster expression ${ }^{1,2}$

$$
k_{F}=k_{\text {rad }}\left(\frac{R_{F}}{R}\right)^{6},
$$

where $\mathrm{k}_{\mathrm{rad}}$ is the radiative rate (typically less than $10^{9} \mathrm{~s}^{-1}$ ), and $R_{F}$ is the well-known Förster radius which is given by the spectral overlap between the fluorescence spectrum of the donor $\left(f_{D}(\omega)\right)$ and the absorption spectrum of the acceptor $\left(\mathrm{a}_{\mathrm{A}}(\omega)\right)$,

$$
\mathrm{R}^{6} \mathrm{~F}=\mathrm{A} \int_{0}^{\infty} \frac{\mathrm{a}_{\mathrm{A}}(\omega) \mathrm{f}_{\mathrm{D}}(\omega) \mathrm{d} \omega}{\omega^{4}},
$$

where A is a numerical constant, determined by, among other things, refractive index, velocity of light. The above Förster expression has been derived on the basis of an assumed Coulombic interaction between the donor and the acceptor and a point dipole approximation of the interaction energy. ${ }^{1-6}$ Usefulness of FRET arises from the strong distance dependence of rate as shown by Eq. 1. The sensitivity of FRET to distance has led to this technique being called a 'spectroscopic ruler'.

Recently, Förster energy transfer mechanism has been implicated in the energy transfer in thin films of conjugated polymers. These systems are organic semiconductors with interesting opto-electronic properties. Host-guest systems comprised of polymer/polymer and polymer/dye blends offer colour tunability and can be used as colour emission in display systems. Recently Cerullo et al. have carried out femto second pump-probe experiments and observed ultra fast energy transfer in the picosecond timescale. ${ }^{7}$ Experiments by Barbara have revealed evidence of an energy funnel in thin films of MEH-PPV where the absorption spectrum is broad, while the emission spectrum is narrow and red shifted. ${ }^{8}$ Particle in a box' model predicts oscillator strength/radiative rate to be proportional to the length of a polymer segment. Resonance energy transfer has also been used in the study of protein folding. 5,6

In the present article we shall discuss distance de- 
pendence of resonance energy transfer via Coulomb mechanism. It was found that the Förster expression of $1 / \mathrm{R}^{6} \mathrm{de}-$ pendence breaks down at short distances. We shall also discuss use of FRET in polymer dynamics and protein folding.

\section{DISTANCE DEPENDENCE OF THE COULOMBIC ENERGY TRANSFER RATE}

In order to explore the validity of the Förster expression for the distance dependence, we have carried out a quantum chemical calculation of the rate between polyfluorence $\left(\mathrm{PF}_{6}\right)$ and triphenyl (TPP). ${ }^{9}$ Here $\mathrm{PF}_{6}$ is the donor and TPP is the acceptor. In Fig. 1 we show the geometry of the donor and the acceptor and also the way separation between them is varied. The calculation of the rate has been carried out by using the Fermi Golden Rule (FGR). We compute the full resonance-Coulomb coupling matrix element, as well as the point-dipole approximation.

Classic semi-empirical Pariser-Parr-Pople (PPP) Hamiltonian is coupled with single configuration interaction (SCI). From the PPP/SCI wave functions, electronic transition energies and transition dipole moments are calculated based on optimized geometries. ${ }^{9}$

In Fig. 2, we show the calculated distance dependence for a given transition. In the same figure we show the comparison with the point dipole approximation, calculated within the same wave functions. The figure shows that the rate deviates from $1 / \mathrm{R}^{6}$ distance dependence at a small separation.

Several comments on the above result are in order. (i) Point dipole approximation and the Förster expression should remain valid when the distance is large compared to the size of the donor-acceptor system. Therefore, in those

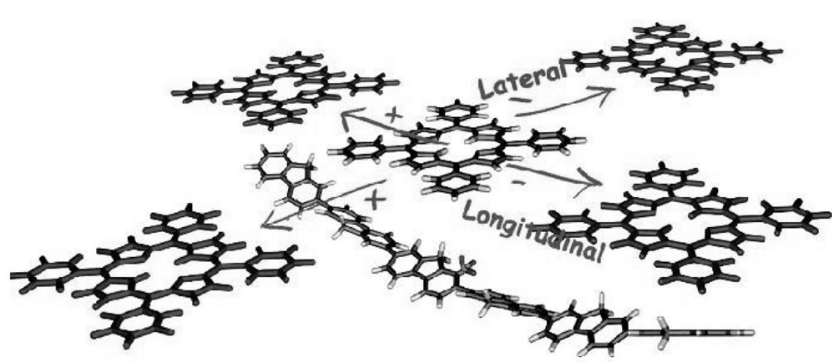

Fig. 1. The geometry of the donor-acceptor system, showing the mode of separation between the pair employed in the calculation. See Ref. 9 for details. applications of FRET where donor and acceptor are dye molecules of molecular dimension say L, Förster expression is expected to remain valid till $\mathrm{R}$ is comparable $L$.

\section{FRET, POLYMER DYNAMICS AND PROTEIN FOLDING}

Fluorescence resonance energy transfer (FRET) is the transfer of the excited state energy from an excited donor to an acceptor. This transfer occurs without the appearance of a photon and results primarily due to the coulombic interaction between a donor and an acceptor. Recently, FRET has emerged as a powerful technique to study both the conformation and the dynamics of polymers and bio-polymers. FRET is also used to monitor the thermodynamic fluctuations and kinetics of protein folding and unfolding reactions.

One can combine FRET with single molecule spectroscopic techniques to obtain histograms of energy transfer efficiency which provides valuable information about the temperature and denaturant dependent conformational states of the protein. ${ }^{10}$

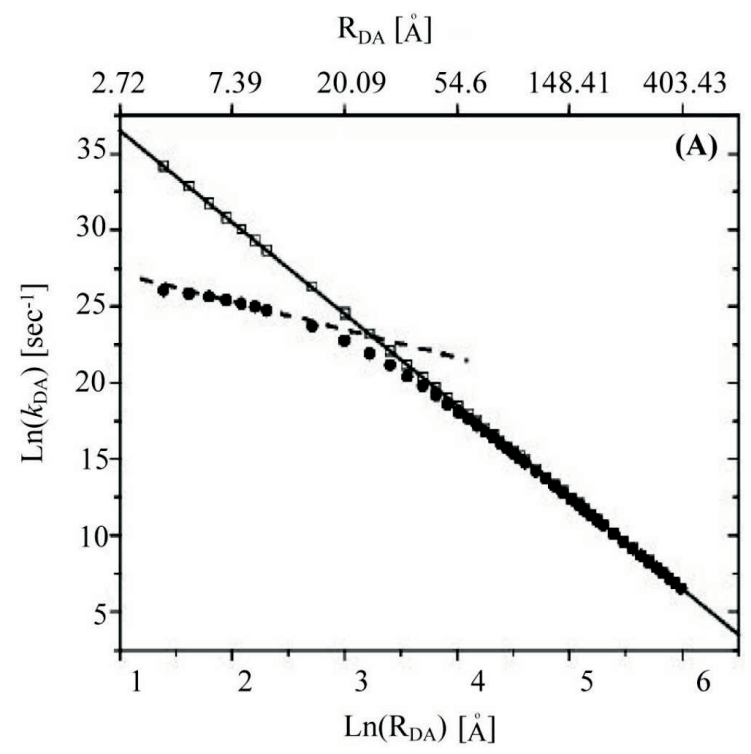

Fig. 2. The calculated distance dependence for a given transition. In the same figure we show the comparison with the point dipole approximation, calculated within the same wave functions. The dashed line shows the deviation from $1 / \mathrm{R}^{6}$ distance dependence at a small separation. See Ref. 9 for details. 


\section{Wilemski-Fixman Theory}

In 1974, Wilemski-Fixman (WF) ${ }^{11}$ presented an elegant theory for the diffusion limited intra-chain reaction of a flexible polymer chain. WF considered a simple Rouse model $^{12}$ to describe the polymer chain with $\mathrm{N}$ monomers. In a Rouse chain, the hydrodynamic and excluded volume interactions are absent.

The complexity of describing the dynamics of energy transfer of polymers in solution arises from the fact that, due to chain connectivity, the Brownian motion of the monomers on the polymer is strongly correlated. The many-body nature of the polymer dynamics can be described by a joint, time dependent probability distribution $P\left(\mathbf{r}^{N}, t\right)$ where $\mathbf{r}^{N}$ denotes the position of all the $N$ polymer beads, at time $t$. The time dependence of the probability distribution $P\left(\mathbf{r}^{N}, t\right)$ can be described by reaction-diffusion equation ${ }^{11}$ as given below.

$$
\frac{\partial}{\partial t} \boldsymbol{P}\left(\mathbf{r}^{N}, t\right)=L_{B} P\left(\mathbf{r}^{N}, t\right)-k_{0} S(R) P\left(\mathbf{r}^{N}, t\right)
$$

where $k_{0} S(R)$ is the sink term and $L_{B}$ is the full $3 N$ dimensional diffusion tensor given as,

$$
\boldsymbol{L}_{B}(\mathbf{r}, \boldsymbol{t})=\boldsymbol{D} \sum_{j=1}^{N} \frac{\partial}{\partial \boldsymbol{r}_{j}} \boldsymbol{P}_{e q}(\mathbf{r}, \boldsymbol{t}) \frac{\partial}{\partial \boldsymbol{r}_{j}} \frac{\boldsymbol{P}(\mathbf{r}, \boldsymbol{t})}{\boldsymbol{P}_{e q}(\mathbf{r}, \boldsymbol{t})}
$$

where the subscript "eq" denotes equilibrium, $R$ is the scalar distance between the two ends of the polymer chain and $D$ is defined as the center of mass diffusion coefficient.

The survival probability, $S_{P}(t)$ is defined as the probability that the chain has not reacted after time $t$ and is given by,

$$
S_{P}(t)=\int P\left(\mathbf{r}^{N}, t\right) d r_{1} d r_{2} \ldots d r_{N}
$$

The Laplace transform of the survival probability is ${ }^{11}$

$$
\hat{S}_{P}(s)=\frac{1}{s}-\frac{k v_{e q}}{s^{2}\left(1+k \hat{D}(s) / v_{e q}\right)},
$$

where $s$ is the Laplace variable, $D(t)$ is the Green's function and $v_{\mathrm{eq}}$ is the equilibrium rate.

WF theory was applied to Rouse chain with Heaviside sink function. This theory provides the understanding of time dependent energy transfer processes because time resolved FRET experiments can be an important tool in understanding the folding/unfolding transitions. Lakshmikanth et al. performed time resolved fluorescence, with the maximum entropy method to analyze the decay kinetics, on a small protein barstar to show the existence of many equilibrium unfolding states contrary to the belief of the two state model of protein folding. ${ }^{13} \mathrm{WF}$ theory with suitable modification can be used to understand protein folding.

\section{FRET Efficiency}

Fluorescence resonance energy transfer (FRET) can serve as a probe to study the structural morphology of a polymer chain. Recently, computational studies of Förster energy transfer efficiency ${ }^{14}$ showed that it can indeed differentiate between different conformational states of collapsed polymer, such as rods, toroids or simply the collapsed disordered state. Experimental studies ${ }^{10}$ demonstrated that the single molecular spectroscopy can be used to obtain the FRET efficiency distribution. FRET efficiency distribution $\left(\Phi_{F}\right)$ is defined by the following relation

$$
\Phi_{F}=\frac{\boldsymbol{k}(\boldsymbol{R})}{\boldsymbol{k}(\boldsymbol{R})+\boldsymbol{k}_{r a d}}
$$

where, $R$ is the distance between donor and acceptor. Assuming the reaction rate to be given by the Förster rate from Eq. 1, we get,

$$
\Phi_{F}=\frac{1}{1+\left(\boldsymbol{R} / \boldsymbol{R}_{F}\right)^{6}},
$$

The probability of FRET efficiency distribution $\boldsymbol{P}\left(\Phi_{F}\right)$ can be defined by the following expression,

$$
\boldsymbol{P}\left(\Phi_{F}\right)=\frac{1}{N} \sum_{i=1}^{N} \delta\left(\Phi_{F}-\Phi_{F}\left(\tau_{r a d}\right)\right)
$$

In each simulation, after choosing a donor-acceptor pair at time $t=0$, the pair is followed till the trajectory gets terminated due to the energy transfer between the donor and acceptor pair. Here, $N$ is the total number of independent polymer chain, and $\tau_{\text {rad }}$ is time taken for a trajectory to terminate from the time of its generation.

\section{COMPARISON BETWEEN WF THEORY AND SIMULATION}

The predictions of WF theory are compared with Brownian dynamics simulations on the Rouse chain model. 
The Rouse chain is a simple polymer model having only bonding interaction and no excluded volume. This is called the ideal chain. Brownian Dynamics (BD) simulations are carried out for an ideal Rouse chain where the neighboring beads interact via a harmonic potential U given by,

$$
\beta U=\frac{3}{2 b^{2}} \sum_{j=1}^{N}\left(r_{j}-r_{j+1}\right)^{2}
$$

where $\beta^{-1}$ is the Boltzmann constant times the temperature, $r_{j}$ is the position vector of bead $\mathrm{j}$, and the number of beads constituting the polymer chain is $N+1$. The mean square bond length is $b^{2}$. The equation of motion of the beads under Brownian motion is given by,

$$
r_{j}(t+\Delta t)=r_{j}(t)+F_{j}(t) \Delta t+\Delta X^{G}(t),
$$

where $r_{j}(t)$ is the position of $\mathrm{j}$-th particle at time $t, F_{j}(t)$ is the systematic force and $\Delta X^{G}(t)$ is the random force obtained from a Gaussian distribution with zero mean and standard deviation of $2 D \Delta t$. In Eq. (11) the time and energy scales are fixed by using units where $\beta=1$, the bead diffusion coefficient $D_{0}=1$, and the mass of bead $m=1$. All the results are presented in these dimensionless units. $\Delta t$ is varied, between 0.0001 and 0.01 , depending on $\mathrm{R}_{\mathrm{F}}$ value. The larger the $R_{F}$ the greater is the requirement for the smaller time

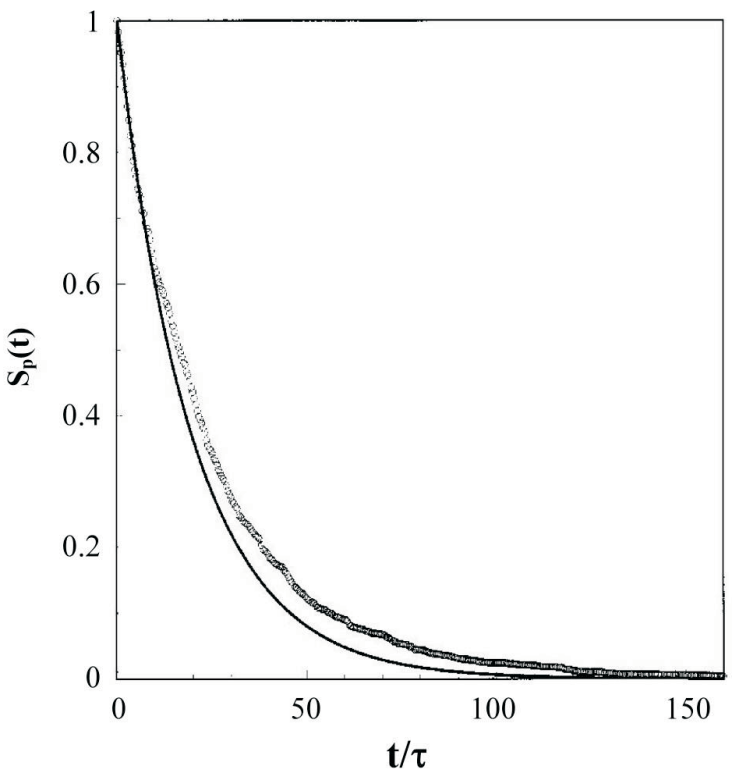

Fig. 3. Comparison of simulation results (symbols) for $S_{P}(t)$ to predictions of the WF theory (line) for a large Förster rate, namely $\mathrm{k}_{\mathrm{F}}=10$ at $\mathrm{R}_{\mathrm{F}}=1$ for $\mathrm{N}$ $=50$. See Refs. $14-15$ for details. step. For example, at $\mathrm{k}_{\mathrm{F}}=1$, when $\mathrm{R}_{\mathrm{F}}=1$ the $\Delta t=0.01$ is employed and for $\mathrm{R}_{\mathrm{F}}=5$, a much smaller time step, $\Delta t=$ 0.0002 is used.

Survival probabilities obtained from Brownian dynamics simulations agree well with the predictions of WF theory. Although the agreement is good for small $\mathrm{R}_{\mathrm{F}}$ values, the simulations results start deviating from WF at larger $\mathrm{R}_{\mathrm{F}}$ values (Srini et al.). Here the comparison between simulation results and WF theory is shown for different Förster rate $\mathrm{k}_{\mathrm{F}}$. Fig. 3 shows the results for large Förster rate while Fig. 4 shows the comparison for small Förster rate, but relatively larger Förster radius.

The decay of survival probability is generally nonexponential, more so when the Förster radius is less than the radius of the polymer. The Wilemski-Fixman theory requires generalization when Förster rate is much faster than the rate of diffusion.

\section{FRET DURING FOLDING OF HP-36}

FRET efficiency measurement on a single molecule provides the insight about the distributions of the chromophores in the protein molecule. ${ }^{10}$ This FRET efficiency distribution, in turn, gives a measure of folding. The experiment by Deniz et al. shows that the FRET efficiency distri-

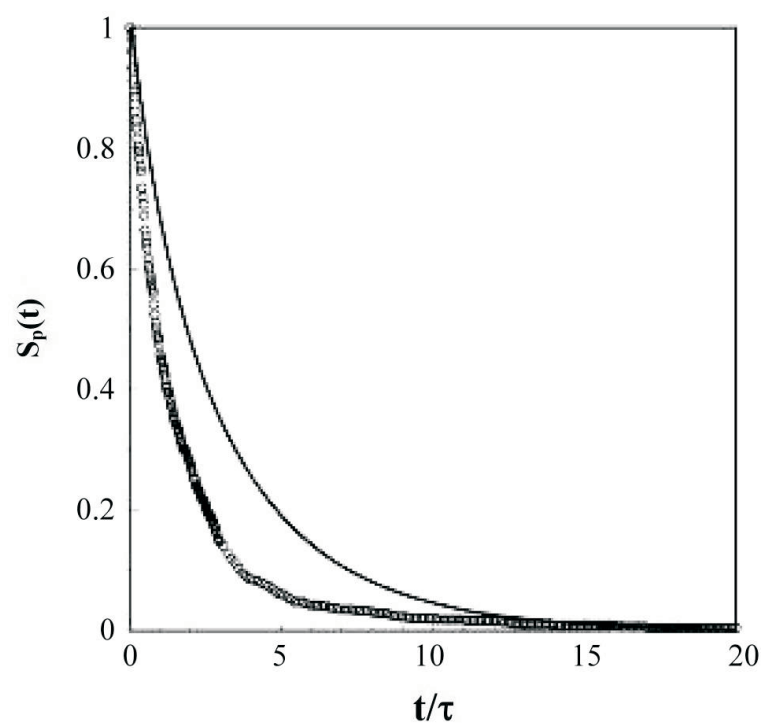

Fig. 4. Comparison of simulation results (symbols) for $S_{P}(t)$ to predictions of the WF theory (line) for a longer chain $(\mathrm{N}=100)$ at $\mathrm{R}_{\mathrm{F}}=8$ and $\mathrm{k}_{\mathrm{F}}=1$. See Refs. 14-15 for details. 
bution has a peak around 1.0 when the protein is folded and when the protein is denatured, the efficiency distribution shifts towards lower FRET efficiency. We find that the results are significantly different in the case of the model protein which shows multistage dynamics, which can potentially be captured by FRET.

Simulations have been carried out in this regard with model protein HP-36. The protein HP-36 is a thermostable subdomain of the chicken villin headpiece and it can fold autonomously to a stable structure. A large number of studies has been carried out on this protein, both using offlattice and all atom models. ${ }^{14-18}$ We have recently presented such a study by using a minimalistic model. ${ }^{16-18}$ Construction of our minimalistic potential is motivated by the hydrophobicity of different amino acids and also the different helical propensity of the amino acid residues. The detail of the model is given elsewhere. ${ }^{14-18}$ The structures obtained after folding resemble, to a good extent, the native state of the real protein. Interestingly, the dynamics shows a multistage folding. The FRET efficiency distribution plotted in the figure below brings out the essential distribu- tion of the separation between the chosen donor-acceptor pair.

FRET has been calculated here along the folding trajectory between different hydrophobic residues. The dynamics of fluorescence resonance energy transfer reflects the multistage folding phenomena. The histogram of the folding efficiency distribution shows interesting features.

Fig. 5 presents the histogram of Förster transfer efficiency $\phi_{F}$ both for the high temperature unfolded and the low temperature folded states of the model protein, with the donor and acceptor placed at 9 th and 35 th side residue atoms both of which are hydrophobic. These distributions have been obtained by calculating FRET efficiency of the equilibrium initial configurations at high temperature and the folded configurations at low temperature. The folded configurations have been generated by quenching the high temperature, extended configuration to the low temperature, collapsed configuration. FRET efficiency shows broad distributions at high temperature as expected. However, the distribution for low temperature folded states is rather interesting. This histogram, although showing a peak

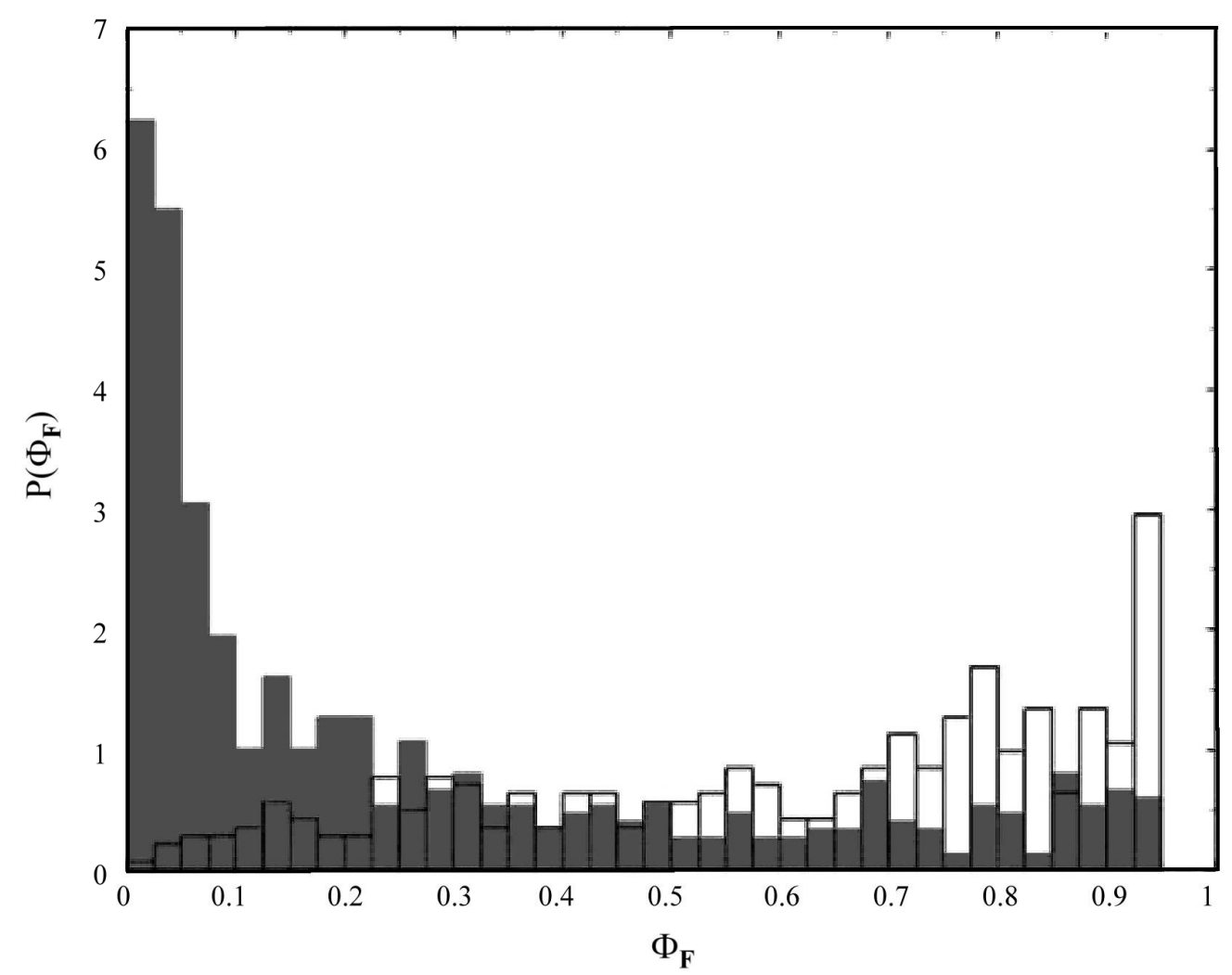

Fig. 5. Probability distribution of FRET efficiency of 9-35 side residue pair is plotted for both high (solid line) and low temperature (dashed line) configurations for $R_{F}=20 \AA$. See Refs. 16-17 for details. 
at high Förster efficiency, does show a broader tail extending up to very low efficiency. Note that the present calculation of this distribution involved a large number (584) of quenched configurations. The significant population at low efficiency shows that many of the quenched states have the donor-acceptor pair separated by a significant distance. This signifies that there are many entangled and misfolded states with the residues under study lying far apart even in the low temperature folded states unlike in the case of homopolymers. This is actually rather different from what has been reported in some experimental studies which provide a reverse description, with a relatively broad peak in the efficiency distribution for the extended states and a sharper distribution for the folded states.

The FRET efficiency $\mathrm{F}_{F}$ depends critically on the value of Förster radius $\mathrm{R}_{F}$ and the nature of donor and acceptor. Protein folding is governed mainly by the hydrophobic force. So change in FRET efficiency distribution is interesting for the hydrophobic pairs because it gives direct information about large scale conformational change. While the broad distribution of the probability in high temperature is expected due to a flat energy surface, the distribution at low temperature signifies the misfolded and trapped states signifying a rather complicated potential energy landscape for this model protein.

Fig. 6 shows the time dependence of survival probability $S_{P}(t)$ in FRET using Förster energy transfer rate (Eq. 1) for the 9-35 pair along the Brownian dynamics trajectory leading to the most stable structure. The Förster radius $R_{F}$ is taken as $10 \AA$. The dynamics of FRET shows initially very slow decrease of $S_{P}(t)$ to be followed by a sudden drop at around 2400 as seen in case of the different dynamical properties discussed below. $S_{P}(t)$ is found to be relatively insensitive to $k_{\text {rad }}$.

The time dependence shown in Fig. 6 can be understood from Fig. 7 where the time variation of the radius of gyration is plotted. The multistage folding process can be easily understood by monitoring the radius of gyration of the model protein with time. Consistent with the dynamics of FRET, there is a long plateau observed at the final stage of folding. This could be correlated with the underlying landscape of the model protein. The long slope could have arisen because of the entropic bottleneck created by an enormous number of conformations at that particular energy level and thus delay the folding process by an enor- mous amount. It is interesting to note that FRET is sensitive to the slow dynamics that occurs after reduced time 2000 and thus to the dynamics of topological pair contact formation. This is due to our choice of $R_{F}=10 \AA$ which is also the plateau value of the radius of gyration in Fig. 6.

\section{CONCLUSION}

In view of the great popularity of fluorescence energy transfer as a spectroscopic ruler to determine structure and dynamics of biopolymers, a critical evaluation of the merits and demerits of Förster energy transfer rate which is often used in the analysis of experimental results is a worthwhile exercise. Recently, FRET has been used in the development of metal based nanosensors which shows quite different distance dependence. ${ }^{19}$

Because the Coulomb coupling depends on the relative orientation of the charge distribution, energy transfer rate also depends on the orientation. Recently a detailed dynamical study of this orientation dependence was carried out. ${ }^{20}$ It was found that if dynamical averaging exists, then the effects of orientation dependence are not paramount. However, in quenched configuration (such as in thin films),

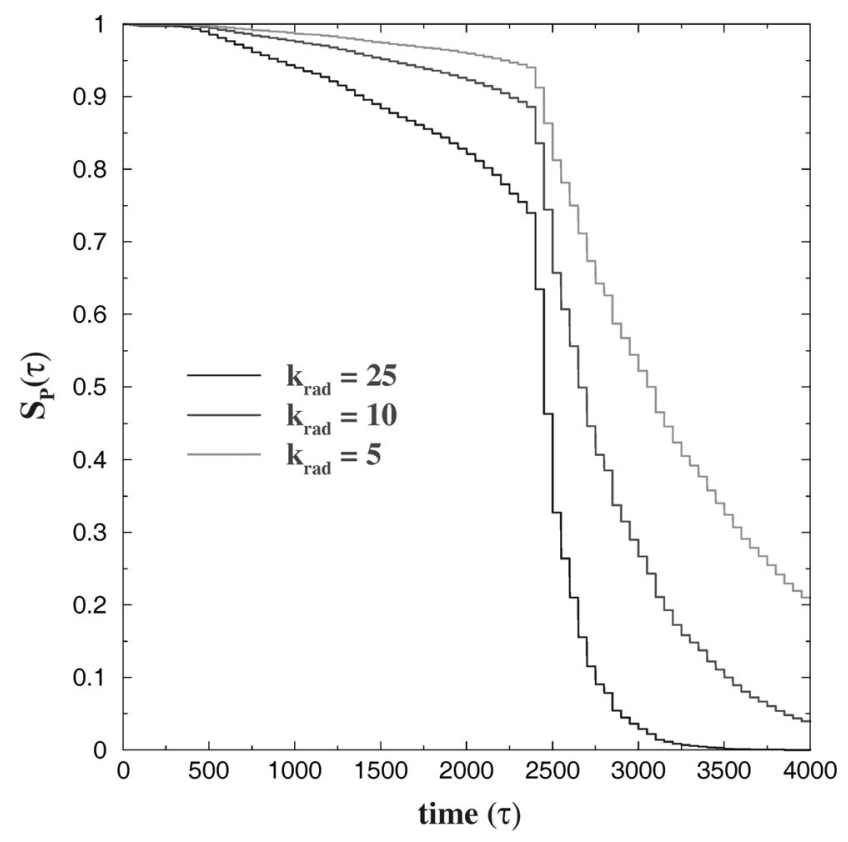

Fig. 6. FRET survival probability of 9-35 side residue pair is shown for different radiative rates at $R_{F}=$ $10 \AA$ A. See Refs. 16-17 for details. 


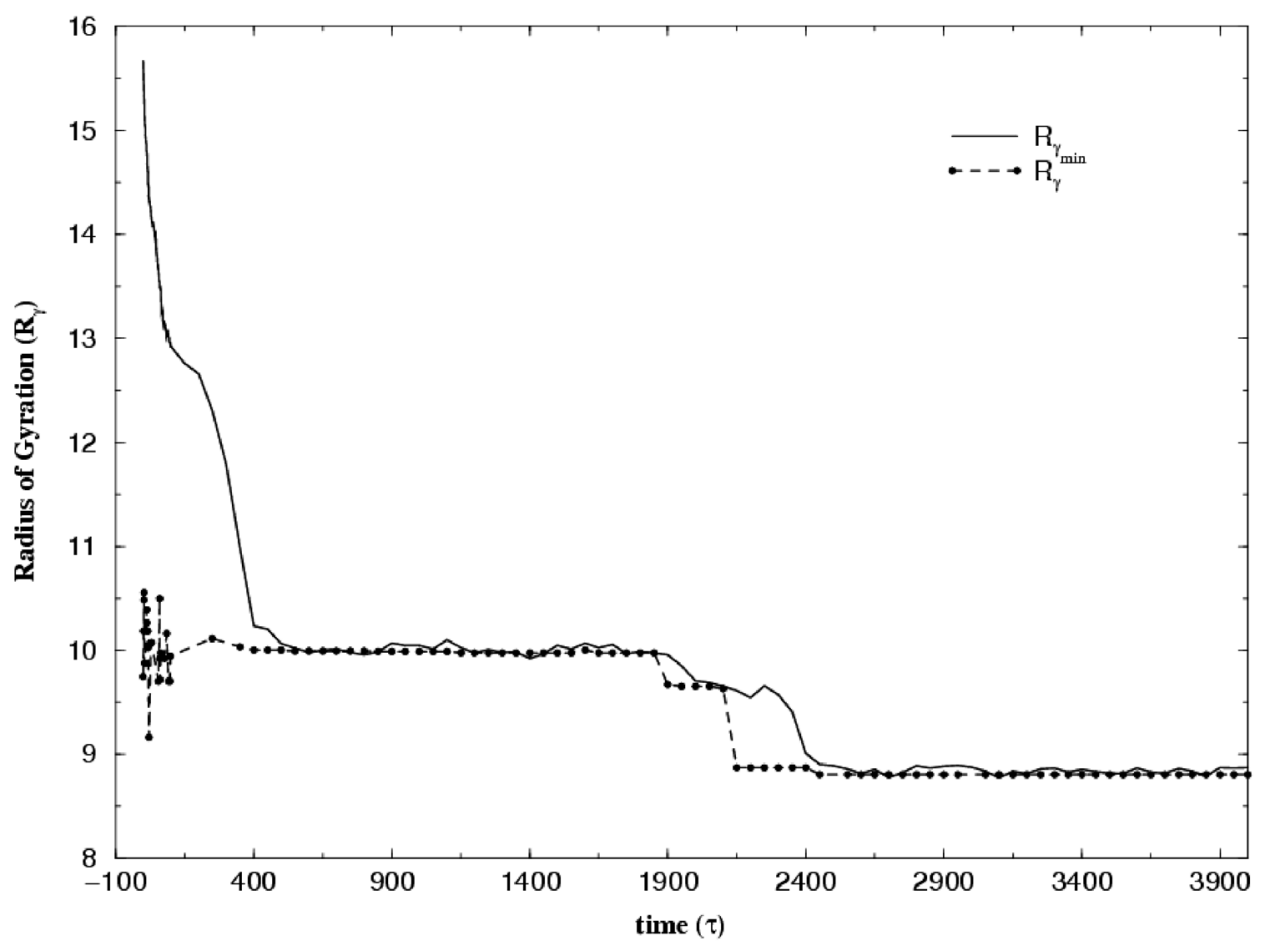

Fig. 7. Dynamics of radius of gyration shows multistage decay. The solid line is the radius of gyration of the model protein and dot-dashed line shows the radius of gyration of the minimized configuration. See. Refs. 16-18 for details.

one must take heed of the relative orientation of the donor-acceptor pair.

\section{ACKNOWLEDGEMENT}

It is a pleasure to thank Dr. G. Srinivas (IISc., Bangalore), Dr. Kim Wong, Professor Peter Rossky and Professor Paul Barbara (UT-Austin, Texas, USA) for collaboration. This work was supported in parts by grants from DST and CSIR.

Received May 5, 2005.

\section{REFERENCES}

1. Förster, Th. Ann. Phys. (Leipzig) 1948, 2, 55.

2. Förster, Th. "Delocalized excitation and excitation transfer" in Modern Quantum Chemistry, Istanbul Lectures, Part III: Action of Light and Organic Crystals; Sinanoglu, O., Ed.;
Academic Press: New York, 1965.

3. Lakowicz, J. R. Principles of Fluorescence Spectroscopy; Plenum: New York, 1983.

4. May, V.; Kuhn, O. Charge and Energy Transfer Dynamics in Molecular Systems; Wiley-VCH, Verlag: Berlin, 2000.

5. Telford, J. R.; Wittung-Stafshede, P.; Gray, H. B.; Winkler, J. R. Acc. Chem. Res. 1998, 31, 755; Pascher, T.; Chesick, J. P.; Winkler, J. R.; Gray, H. B. Science 1996, 271, 1558.

6. Lyubovitsky, J. G.; Gray, H. B.; Winkler, J. R. J. Am. Chem. Soc. 2002, 124, 5481.

7. Cerullo, G.; Stagira, G.; Zavelani-Rossi, M.; Silvestri, S.; Virgili, T.; Lidzey, D. G.; Bradley, D. D. C. Chem. Phys. Lett. 2001, 335, 27-29.

8. Hu, D.; Yu, J.; Barbara, P. F. J. Am. Chem. Soc., 1999, 121, 6936-6937.

9. Wong, K. F.; Bagchi, B.; Rossky, P. J. J. Phys. Chem. A, 2004, 108, 5752-5763.

10. Deniz, A. A.; Laurence, T. A.; Beligere, G. S.; Dahan, M.; Martin, A. B.; Chemla, D. S.; Dawson, P. E.; Schultz, P. G.; Weiss, S. Proc. Natl. Acad. Sci. USA 2000, 97, 5179.

11. Wilemski, G.; Fixman, M. J. Chem. Phys. 1974, 60, 866; ibid 1974, 878.

12. Rouse, P. E. J. Chem. Phys. 1953, 21, 1272.

13. Lakshmikanth, G. S.; Sridevi, K.; Krishnamoorthy, G.; 
Jayant, B. Udgaonkar, 2001, 8, 799.

14. Srinivas, G.; Bagchi, B. J. Chem. Phys. 2002, 116, 837.

15. Srinivas, G.; Yethiraj, A.; Bagchi, B. J. Phys. Chem. B 2001, 105,2475 .

16. Mukherjee, A.; Bagchi, B. J. Chem. Phys. 2003, 118, 4733.
17. Mukherjee, A.; Bagchi, B. J. Chem. Phys. 2004, 120, 1602

18. Srinivas, G.; Bagchi, B. Phys. Chem. Comm. 2002, 5, 59.

19. Yun, C. S. et al. J. Am. Chem. Soc. 2005, 127, 3115-3119.

20. Srinivas, G.; Bagchi, B. J. Phys. Chem. B 2001, 105, 9370. 